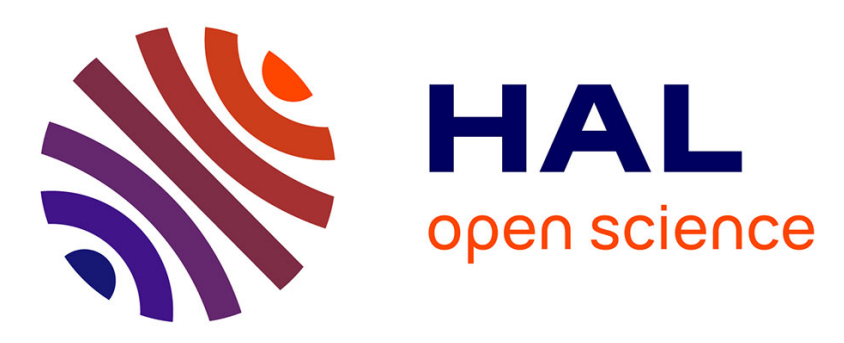

\title{
An Algebraic Approach to Continuous Collision Detection for Ellipsoids
}

Xiaohong Jia, Yi-King Choi, Bernard Mourrain, Wenping Wang

\section{To cite this version:}

Xiaohong Jia, Yi-King Choi, Bernard Mourrain, Wenping Wang. An Algebraic Approach to Continuous Collision Detection for Ellipsoids. Computer Aided Geometric Design, 2011, 28 (3), pp.164-176. 10.1016/j.cagd.2011.01.004 . hal-00646511

HAL Id: hal-00646511

https://hal.inria.fr/hal-00646511

Submitted on 30 Nov 2011

HAL is a multi-disciplinary open access archive for the deposit and dissemination of scientific research documents, whether they are published or not. The documents may come from teaching and research institutions in France or abroad, or from public or private research centers.
L'archive ouverte pluridisciplinaire HAL, est destinée au dépôt et à la diffusion de documents scientifiques de niveau recherche, publiés ou non, émanant des établissements d'enseignement et de recherche français ou étrangers, des laboratoires publics ou privés. 


\title{
An Algebraic Approach to Continuous Collision Detection for Ellipsoids
}

\author{
Xiaohong Jia ${ }^{\mathrm{a}, *}$, Yi-King Choi ${ }^{\mathrm{a}}$, Bernard Mourrain ${ }^{\mathrm{b}}$, Wenping Wang ${ }^{\mathrm{a}}$ \\ ${ }^{a}$ The University of Hong Kong, Pokfulam Road, Hong Kong, China \\ ${ }^{b}$ GALAAD, INRIA Méditerranée, 2004 route des lucioles, 06902 Sophia-Antipolis, France
}

\begin{abstract}
We present algebraic conditions for characterizing three configurations formed by two ellipsoids in $\mathbb{R}^{3}$ that are relevant to collision detection: separation, external touching and overlapping. These conditions are given in terms of explicit formulae expressed by the subresultant sequence of the characteristic polynomial of the two ellipsoids and its derivative. For any two ellipsoids, the signs of these formulae can easily be evaluated to classify their configuration. Furthermore, based on these algebraic conditions, an efficient method is developed for continuous collision detection for two moving ellipsoids under arbitrary motion.

Keywords: moving ellipsoids, characteristic equation, continuous collision detection, algebraic conditions, subresultants
\end{abstract}

\section{Introduction}

Collision detection finds many applications in computer graphics, computer animation, CAD/CAM as well as computational physics (see Eberly (2001)). Since collision detection for general freeform moving objects is computationally very expensive, bounding volumes are often used to approximate the freeform objects in order to reduce computational cost. Ellipsoids are good candidates for such bounding volumes since they have low algebraic degree and are tight bounding volumes for a wide class of objects (Bouville (1985); Lu et al. (2007)). Minimum bounding (or enclosing) ellipsoids and their computations have long been studied as a classical mathematical problem, see e.g., Welzl (1991); Kumar and Yildirim (2005); Todd and Yildirim (2007); Schröker (2008), and have found important applications to not only CAGD and computer graphics but also other areas such as data uncertainty analysis.

Collision detection for ellipsoids has been an active research topic over the past years, see Ju et al. (2001); Rimon and Boyd (1997); Shiang et al. (2000); Wang et al. (2004); Choi et al. (2006, 2009). Wang et al. (2001) provide algebraic conditions for the three important configurations, i.e., separation, external touching and overlapping, of two static ellipsoids in $\mathbb{R}^{3}$, the three dimensional affine space. It is shown that the relative

\footnotetext{
* Corresponding author

Email addresses: xhjia@cs.hku.hk (Xiaohong Jia), ykchoi@cs.hku.hk (Yi-King Choi), mourrain@sophia.inria.fr (Bernard Mourrain), wenping@cs.hku.hk (Wenping Wang) 
position of two ellipsoids is related to the root pattern of their characteristic equation. Specifically, two ellipsoids are separate if and only if their characteristic equation has two distinct positive roots; and they touch each other externally if and only if there is one positive double root. This result allows us to compute the configuration of two ellipsoids by simply determining the number of the positive roots of a polynomial, which can be derived by the classical Decart's rule and the modified sign variation number of the signed subresultant sequence (e.g., see Basu et al. (2006)).The algebraic conditions given by Wang et al. (2001) lays the theoretical foundation for the follow-up practical applications in collision detection for ellipsoids.

Collision detection mostly deals with moving objects. When the positions of the two ellipsoids are given in a sequence of discrete time frames, which is often the situation in computer animation, temporal and geometric coherence are exploited in Wang et al. (2004) for speeding up the computations. A separating plane for two non-intersecting ellipsoids at a frame is calculated which can then be used to quickly identify if the ellipsoids are still separate or not in the next frame. The more computationally intensive ellipsoidellipsoid intersection test needs to be carried out only when the separating plane fails to guarantee separation of the ellipsoids.

Continuous collision detection (CCD) in which object motions are given by continuous funcions of a time parameter $t$ has been gaining increasing interests in the past decade (see e.g., Redon et al. (2002); Teschner et al. (2005)). It focuses on determining the collision status of objects over a specific time span and is exact in the sense that no discretization of the time domain is needed. For CCD of two moving ellipoids with continuous motions, the method in Wang et al. (2004) mentioned above cannot easily be extended to deal with the problem since the computation of a separating plane requires solving for the roots of the characteristic equation. Based on the algebraic condition by Wang et al. (2001), Choi et al. (2003) reduces the problem to an analysis of the zero set of the characteristic equation which is a bivariate polynomial equation; however, their algorithm involves the brute-force computation of the zero set and is therefore slow. Later Choi et al. (2009) use the same CCD algebraic formulation, and further develop an efficient search scheme to determine the collision time instants from the characteristic equation in real time. The basis idea is to find the contact time instants by alternative searches in the two parameter domains of the characteristic equation. Unfortunately, the use of Bézier clipping in the search means that this method is only applicable to ellipsoids whose motions are expressed as rational functions.

In this paper, we aim at establishing algebraic conditions, based on those by Wang et al. (2001), which are suitable for direct application to CCD of moving ellipsoids under arbitrary motions (such as the commonly used helical motions as in Example 5.2). Our algebraic conditions are based on five explicit formulae derived from the subresultant sequences of the characteristic polynomial of two ellipsoids and its derivative. Essentially, these conditions distinguish the different root patterns of the characteristic equation, therefore distinguishing the configurations formed by two ellipsoids, without having to solve for the roots. We also develop an algorithm for CCD of two moving ellipsoids under arbitrary continuous motions which are not 
necessarily rational. In this case, the formulae will directly lead to five functions in the time parameter $t$, and CCD can simply be done by solving these five functions.

We note that Gonzalez-Vega and Mainar (2008) translate the algebraic conditions in Wang et al. (2001) to a set of closed form formulae to characterize the separation of two ellipsoids, and their results are also based on the subresultant sequence of the characteristic polynomial and its first derivative. However, their conditions do not distinguish the two conditions of external touching and overlapping. Our present work is inspired by Gonzalez-Vega and Mainar (2008) and improves it by further distinguishing external touching and overlapping. The distinction of these two configurations is not only useful in collision detection but also of theoretical interest in its own right.

The remainder of the paper is organized as follows. In Section 2 we review the algebraic conditions given by Wang et al. (2001) and present the algebraic tool of subresultant sequences which will later be used to derive our explicit formulae. We then derive in Section 3 the five explicit formulae for distinguishing the root patterns of the characteristic equation, thus characterizing the configurations of two ellipsoids. In Section 4, we examine the computational cost by optimizing the evaluation of the five explicit formulae. In Section 5 , we present examples on applying our method to continuous collision detection for ellipsoids and compare the efficiency of our approach with Choi et al. (2009). We conclude the paper in Section 6 .

\section{Preliminaries}

Given two ellipsoids $\mathcal{A}: X^{T} A X=0$ and $\mathcal{B}: X^{T} B X=0$ in $\mathbb{R}^{3}$, where $X=(x, y, z, w)^{T}$ are the homogeneous coordinates of points $\left(\frac{x}{w}, \frac{y}{w}, \frac{z}{w}\right) \in \mathbb{R}^{3}$ and $A, B$ are $4 \times 4$ coefficient matrices with elements in $\mathbb{R}$, the characteristic polynomial of the ellipsoids $\mathcal{A}$ and $\mathcal{B}$ is defined by

$$
f(\lambda)=\operatorname{det}(\lambda A+B)
$$

The characteristic polynomial $f(\lambda)$ has degree 4 in $\mathbb{R}[\lambda]$, where $\mathbb{R}[\lambda]$ is the polynomial ring with real coefficients. Since $A$ and $B$ represent ellipsoids, we have $\operatorname{det}(A)<0$ and $\operatorname{det}(B)<0$; hence dividing each coefficient of $f$ by the leading coefficient $\operatorname{det}(A)$, we get the monic form of $f$ :

$$
f(\lambda)=\lambda^{4}+a \lambda^{3}+b \lambda^{2}+c \lambda+d,
$$

where $d=\operatorname{det}(B) / \operatorname{det}(A)>0$. It also follows that zero cannot be a root of $f(\lambda)=0$. We further assume that the interiors of the ellipsoids $\mathcal{A}$ and $\mathcal{B}$ are defined by $X^{T} A X<0$ and $X^{T} B X<0$.

Now we define the three configurations of two ellipsoids: separate, external touching and overlapping. An ellipsoid is regarded as a solid bounded by the boundary surface $X^{T} A X=0$. Two ellipsoids are separate if their boundary surfaces and interiors share no common points; otherwise, they are said to intersect. 
Furthermore, two intersecting ellipsoids are said to overlap if their interiors have a common point; otherwise they touch externally. That is, two intersecting ellipsoids may overlap or touch externally.

The following algebraic conditions are given by Wang et al. (2001) on the configurations of two ellipsoids $\mathcal{A}$ and $\mathcal{B}$.

Theorem 2.1. Wang et al. (2001)

1. The characteristic equation $f(\lambda)=0$ always has at least two negative roots;

2. The two ellipsoids $\mathcal{A}$ and $\mathcal{B}$ are separate if and only if $f(\lambda)=0$ has two distinct positive roots;

3. The two ellipsoids $\mathcal{A}$ and $\mathcal{B}$ touch each other externally if and only if $f(\lambda)=0$ has a positive double root;

4. The two ellipsoids $\mathcal{A}$ and $\mathcal{B}$ overlap if and only if $f(\lambda)=0$ has no positive root.

We shall derive explicit expressions whose signs indicate the root pattern as described in Theorem 2.1. Such a symbolic approach avoids computing the roots of the characteristic equation and makes it possible to develop an efficient method for continuous collision of two moving ellipsoids when there is a time parameter involved. Note that a symbolic treatment has been proposed for two ellipses in Choi et al. (2006), which provides a basis for continuous collision detection for two moving ellipses therein. However, it is a more difficult problem for two ellipsoids because, unlike the case of ellipses, the appearance of a double root of the characteristic equation does not necessarily mean any configuration change for two ellipsoid. See Choi et al. (2006) for a brief discussion about this difficulty.

We now introduce the concept of the subresultant sequence, an algebraic tool to be used in our derivation. For more details, see for example, Geddes et al. (1992), Basu et al. (2006), von zur Gathen and Gerhard (1999), Kerber (2009).

Definition 2.2. Basu et al. (2006) Let

$$
f(\lambda)=\sum_{k=0}^{n} p_{k} \lambda^{k}, g(\lambda)=\sum_{k=0}^{m} q_{k} \lambda^{k}
$$

be two polynomials in $\mathbb{R}[\lambda]$ with degrees $n=\operatorname{deg}(f) \geq \operatorname{deg}(g)=m$. The $i$-th Sylvester-Habicht matrix of $f$ 
and $g$, denoted by $\operatorname{SyHa}_{i}(f, g)$, is defined by

$$
\operatorname{SyHa}_{i}(f, g):=\left(\begin{array}{cccccccc}
p_{n} & \cdots & \cdots & \cdots & \cdots & p_{0} & 0 & 0 \\
0 & \ddots & & & & & \ddots & 0 \\
\vdots & \ddots & p_{n} & \cdots & \cdots & \cdots & \cdots & p_{0} \\
\vdots & & 0 & q_{m} & \cdots & \cdots & \cdots & q_{0} \\
\vdots & . & . \cdot & & & & . \cdot & 0 \\
0 & . \cdot & & & & . \cdot & . \cdot & \vdots \\
q_{m} & \cdots & \cdots & \cdots & q_{0} & 0 & \cdots & 0
\end{array}\right) \quad\{n-i
$$

The $i$-th signed subresultant $\mathbf{s r}_{i}(\lambda)$ of $f$ and $g$ is the determinant of the $(n+m-2 i) \times(n+m-2 i)$ matrix whose first $n+m-2 i-1$ columns are taken from the first $n+m-2 i-1$ columns of $\operatorname{SyHa}_{i}(f, g)$, and the last column is the polynomial sequence $\lambda^{n-i-1} f, \lambda^{n-i-2} f, \cdots, f, g, \lambda g, \cdots, \lambda^{m-i-1} g$. Note that the first signed subresultant $\mathbf{s r}_{0}(\lambda)$ is equal to the resultant $\operatorname{Res}(f, g)$ of $f$ and $g$.

In the sequel, we consider the subresultant sequence of a polynomial $f$ and its first derivative $f^{\prime}$. Denote the coefficient of the degree $j$ term of the polynomial $\mathbf{s r}_{i}$ by $\mathbf{s r}_{i j}, j=0, \cdots, \operatorname{deg}\left(\mathbf{s r}_{i}\right)$. The following property of subresultant sequence will be important to our later analysis.

Lemma 2.3. Let $f(\lambda) \in \mathbb{R}[x]$. Then $\operatorname{deg}\left(\operatorname{gcd}\left(f(\lambda), f^{\prime}(\lambda)\right)\right)=k$ if and only if $\mathbf{s r}_{k k} \neq 0$ and $\mathbf{s r}_{i i} \equiv 0, i=$ $0, \cdots, k-1$. Furthermore, we have $\mathbf{s r}_{k}(\lambda)=\operatorname{gcd}\left(f(\lambda), f^{\prime}(\lambda)\right)$.

\section{Explicit Formulae for Configurations of Two Ellipsoids}

In this section we shall derive explicit formulae for characterizing the configurations of two static ellipsoids. Throughout we shall repeatedly apply the classical Descartes' rule of signs and the modified sign variations of the signed subresultants. See the details in, for example, Basu et al. (2006).

Proposition 3.1 (Descartes' rule of signs). Let $f(\lambda)=a_{n} \lambda^{n}+\cdots+a_{0} \in \mathbb{R}[\lambda]$. Then the number of positive roots of $f(\lambda)=0$ is equal to $\operatorname{Var}\left(a_{n}, \cdots, a_{0}\right)-2 k$ for some non-negative integer $k$, where $\operatorname{Var}(s)$ is the number of sign variations in a sequence $s$.

Corollary 3.2. The number of negative roots of $f(\lambda)=0$ is equal to $\left.\operatorname{Var}\left((-1)^{n} a_{n},(-1)^{n-1} a_{n-1}, \cdots, a_{0}\right)\right)-$ $2 k$ for some non-negative integer $k$.

Let $f(\lambda)=\lambda^{4}+a \lambda^{3}+b \lambda^{2}+c \lambda+d$ be the characteristic polynomial of two ellipsoids $\mathcal{A}$ and $\mathcal{B}$. Then we have the following lemma.

Lemma 3.3. $\operatorname{Var}(1, a, b, c, d) \neq 4$. 
Proof. Suppose on the contrary that $\operatorname{Var}(1, a, b, c, d)=4$. Then we shall have $\operatorname{Var}(1,-a, b,-c, d)=0$, which by Corollary 3.2 means that the characteristic equation $f(\lambda)=0$ has no negative root. This contradicts Theorem 2.1.

Lemma 3.4. If $\operatorname{Var}(1, a, b, c, d)=0$, the two ellipsoids $\mathcal{A}$ and $\mathcal{B}$ overlap.

Proof. By Descartes' rule of signs, $\operatorname{Var}(1, a, b, c, d)=0$ implies that the characteristic equation $f(\lambda)=0$ has no positive root. By Theorem 2.1, the two ellipsoids overlap.

Hence given the characteristic polynomial $f(\lambda)$ of two static ellipsoids, we can immediately tell that the two ellipsoids overlap if $\operatorname{Var}(1, a, b, c, d)=0$. However, the converse of Lemma 3.4 is not true.

Lemma 3.5. If two ellipsoids $\mathcal{A}$ and $\mathcal{B}$ overlap, then $\operatorname{Var}(1, a, b, c, d)=0$ or 2 ; further if their characteristic equation $f(\lambda)=0$ has four real roots, then $\operatorname{Var}(1, a, b, c, d)=0$.

Proof. Since the ellipsoids $\mathcal{A}$ and $\mathcal{B}$ overlap, $f(\lambda)=0$ has no positive root. By Descartes' rule of signs and Lemma 3.3, $\operatorname{Var}(1, a, b, c, d)=0$ or 2.

Now if $f(\lambda)=0$ has four real roots, since none of these roots can be positive, we have $\operatorname{Var}(1,-a, b,-c, d)=$ 4 and therefore $\operatorname{Var}(1, a, b, c, d)=0$.

Using the above lemmas, we have

Lemma 3.6. Two ellipsoids $\mathcal{A}$ and $\mathcal{B}$ are separate or externally touch if and only if their characteristic equation $f(\lambda)=0$ has four real roots and $\operatorname{Var}(1, a, b, c, d)=2$.

Proof. " $\Longrightarrow$ ": By Theorem 2.1, the configuration of separation or external touch suggests the characteristic equation has four real roots. On the other hand, By Proposition 3.1, $\operatorname{Var}(1, a, b, c, d)$ is an even number, hence can be 0,2 or 4 . But by Lemma $3.3 \operatorname{Var}(1, a, b, c, d) \neq 4$, and by Lemma $3.4 \operatorname{Var}(1, a, b, c, d) \neq 0$. So $\operatorname{Var}(1, a, b, c, d)=2$, and therefore $f(\lambda)=0$ must have four real roots.

" $\Longleftarrow$ : Since $\operatorname{Var}(1, a, b, c, d)=2$, by Proposition $3.1 f(\lambda)=0$ has zero or two positive roots. If $f(\lambda)=0$ has no positive root, the two ellipsoids overlap, which by Lemma 3.5 yields $\operatorname{Var}(1, a, b, c, d)=0$ and hence a contradiction. Therefore $f(\lambda)=0$ has two positive roots, that is, the two ellipsoids are either separate or touch externally.

Table 1 summarizes Lemma 3.3 to Lemma 3.6.

Next we are going to show how to determine whether the characteristic equation $f(\lambda)=0$ has four real roots. Consider the characteristic polynomial $f(\lambda)$ and its derivative $f^{\prime}(\lambda)$, together with their first three subresultants $\mathbf{s r}_{0}(\lambda), \mathbf{s r}_{1}(\lambda), \mathbf{s r}_{2}(\lambda)$. Denote the sequence

$$
\mathcal{P}=f(\lambda), f^{\prime}(\lambda), \mathbf{s r}_{2}(\lambda), \mathbf{s r}_{1}(\lambda), \mathbf{s r}_{0}
$$


Table 1: Configurations of two ellipsoids and the corresponding values for $\operatorname{Var}(1, a, b, c, d)$ and the number of real roots for $f(\lambda)=0$.

\begin{tabular}{|c|c|c|}
\hline $\operatorname{Var}(1, a, b, c, d)$ & \# of real roots for $f(\lambda)=0$ & Configuration of two ellipsoids \\
\hline 0 & 2 or 4 & overlap \\
\hline \multirow{2}{*}{2} & 4 & separate or externally touch \\
\cline { 2 - 3 } & 2 & overlap \\
\hline 4 & \multicolumn{2}{|c|}{ impossible } \\
\hline
\end{tabular}

where by Definition 2.2,

$$
\begin{aligned}
f(\lambda) & =\lambda^{4}+a \lambda^{3}+b \lambda^{2}+c \lambda+d \\
f^{\prime}(\lambda) & =4 \lambda^{3}+3 a \lambda^{2}+2 b \lambda+c \\
\mathbf{s r}_{2}(\lambda) & =\left(-8 b+3 a^{2}\right) \lambda^{2}+(2 a b-12 c) \lambda+a c-16 d \\
\mathbf{s r}_{1}(\lambda) & =\left(-6 a^{3} c+2 a^{2} b^{2}-12 a^{2} d+28 a b c-8 b^{3}-36 c^{2}+32 b d\right) \lambda \\
& -9 a^{3} d+a^{2} b c+3 a c^{2}+32 a b d-4 b^{2} c-48 c d \\
\mathbf{s r}_{0} & =-192 c d^{2} a+256 d^{3}+144 c^{2} d b+b^{2} a^{2} c^{2}-6 c^{2} d a^{2}+18 c^{3} b a \\
& +144 b a^{2} d^{2}-4 b^{3} a^{2} d+16 b^{4} d-4 c^{3} a^{3}-128 d^{2} b^{2}-4 b^{3} c^{2}-27 a^{4} d^{2} \\
& -80 c b^{2} a d+18 c b a^{3} d-27 c^{4} .
\end{aligned}
$$

Using the same notation as in Basu et al. (2006), let $\operatorname{MVar}(\mathcal{P} ; a)$ denote the modified ${ }^{1}$ number of sign variations in a sequence of polynomials $\mathcal{P}=P_{0}, \ldots, P_{n}$ evaluated at $a \in \mathbb{R} \cup\{-\infty,+\infty\}$, that is, $\operatorname{MVar}(\mathcal{P} ; a)=\operatorname{MVar}\left(P_{0}(a), \ldots, P_{n}(a)\right)$. Also, denote $\operatorname{MVar}(\mathcal{P} ; a, b)=\operatorname{MVar}(\mathcal{P} ; a)-\operatorname{MVar}(\mathcal{P} ; b)$, where $a, b \in \mathbb{R} \cup\{-\infty,+\infty\}$.

Proposition 3.7. Basu et al. (2006) Let $f$ be a polynomial of degree $n$ in $\mathbb{R}[\lambda]$, and let $a<b$ be elements in $\mathbb{R} \cup\{-\infty,+\infty\}$ that are not roots of $f$. Denote by $\mathcal{P}$ the signed subresultant sequence $\left\{f, f^{\prime}, \mathbf{s r}_{n-2}\left(f, f^{\prime}\right), \cdots, \mathbf{s r}_{0}\left(f, f^{\prime}\right)\right\}$. Then the number of roots of $f$ counting without multiplicities in $\mathbb{R}$ is equal to $\operatorname{MVar}(\mathcal{P} ; a, b)$.

Lemma 3.8. The characteristic equation $f(\lambda)=0$ has four real roots if and only if one of the following four cases holds.

1. $\mathbf{s r}_{22}>0, \mathbf{s r}_{11}>0, \mathbf{s r}_{0}>0$; this occurs if and only if $f(\lambda)=0$ has four distinct simple roots.

2. $\mathbf{s r}_{22}>0, \mathbf{s r}_{11}>0, \mathbf{s r}_{0}=0$; this occurs if and only if $f(\lambda)=0$ has one double root and two distinct simple roots.

\footnotetext{
${ }^{1}$ We first delete those polynomials identical to zero in the sequence $\mathcal{P}$. Then the modified number of sign variations $\operatorname{MVar}(\mathcal{P}, a)$ is similarly defined as the commonly used number of sign variations $\operatorname{Var}(\mathcal{P}, a)$ except that the case $\{+, 0,0,+\}$ or $\{-, 0,0,-\}$ (exactly two zeros between the two nonzero number) counts the variation for two but not zero. See page 330 of Basu et al. (2006) for details.
} 
3. $\mathbf{s r}_{22}>0, \mathbf{s r}_{11}=0, \mathbf{s r}_{0}=0$; this occurs if and only if $f(\lambda)=0$ has two double roots or a simple and a triple root.

4. $\mathbf{s r}_{22}=0, \mathbf{s r}_{11}=0, \mathbf{s r}_{0}=0$; this occurs if and only if $f(\lambda)=0$ has one quadruple root.

Proof. The above enumeration covers all possible cases under which the characteristic equation $f(\lambda)=0$ has four real roots. We now establish the corresponding algebraic conditions for these cases.

For case 1 , by Proposition 3.7, $\operatorname{MVar}(\mathcal{P} ;-\infty,+\infty)=4$. Note that

$$
\begin{aligned}
& \operatorname{MVar}(\mathcal{P} ;-\infty)=\operatorname{MVar}\left(+,-, \mathbf{s r}_{22},-\mathbf{s r}_{11}, \mathbf{s r}_{0}\right) \\
& \operatorname{MVar}(\mathcal{P} ;+\infty)=\operatorname{MVar}\left(+,+, \mathbf{s r}_{22}, \mathbf{s r}_{11}, \mathbf{s r}_{0}\right)
\end{aligned}
$$

Hence the only possible choice is that $\mathbf{s r}_{22}>0, \mathbf{s r}_{11}>0$ and $\mathbf{s r}_{0}>0$.

For case 2 , since $f(\lambda)=0$ has a double root, by Lemma 2.3 we have $\mathbf{s r}_{0}=0$. Again by Proposition 3.7, $\operatorname{MVar}(\mathcal{P} ;-\infty,+\infty)=3$. Note that

$$
\begin{aligned}
& \operatorname{MVar}(\mathcal{P} ;-\infty)=\operatorname{MVar}\left(+,-, \mathbf{s r}_{22},-\mathbf{s r}_{11}, 0\right) \\
& \operatorname{MVar}(\mathcal{P} ;+\infty)=\operatorname{MVar}\left(+,+, \mathbf{s r}_{22}, \mathbf{s r}_{11}, 0\right)
\end{aligned}
$$

Hence the only possible choice is that $\mathbf{s r}_{22}>0, \mathbf{s r}_{11}>0$.

For case 3 , since $f(\lambda)=0$ has two double roots or a simple and a triple root, the degree of $\operatorname{gcd}\left(f(\lambda), f^{\prime}(\lambda)\right)$ is 2. By Lemma 2.3, we have $\mathbf{s r}_{0}=0$ and $\mathbf{s r}_{11}=0$. By Proposition 3.7, $\operatorname{MVar}(\mathcal{P} ;-\infty,+\infty)=2$. Note that

$$
\begin{aligned}
& \operatorname{MVar}(\mathcal{P} ;-\infty)=\operatorname{MVar}\left(+,-, \mathbf{s r}_{22}, 0,0\right) \\
& \operatorname{MVar}(\mathcal{P} ;+\infty)=\operatorname{MVar}\left(+,+, \mathbf{s r}_{22}, 0,0\right)
\end{aligned}
$$

Hence the only possible choice here is that $\mathbf{s r}_{22}>0$.

For case 4 , by Lemma $2.3, f(\lambda)=0$ has one quadruple root if and only if $\mathbf{s r}_{22}=\mathbf{s} \mathbf{r}_{11}=\mathbf{s r}_{0}=0$.

Next we shall provide algebraic conditions for characterizing the configurations of two ellipsoids.

Lemma 3.9. Let $f(\lambda)=0$ be the characteristic equation of two ellipsoids $\mathcal{A}: X^{T} A X=0$ and $\mathcal{B}: X^{T} B X=$ 0 . If $\mathbf{s r}_{22} \leq 0$ then the two ellipsoids overlap.

Proof. If $\mathbf{s r}_{22} \leq 0$, then $\operatorname{MVar}(\mathcal{P} ;-\infty,+\infty) \leq 2$. By Proposition 3.7, $f(\lambda)=0$ has at most 2 distinct real roots. As $f(\lambda)=0$ has at least two negative roots (counted with multiplicity), considering $f(-\infty)>0$, $f(+\infty)>0$ and $f(0)=d>0, f$ has either no positive roots or two double roots of opposite sign. For the latter case, we must have both $\operatorname{MVar}(\mathcal{P} ;-\infty,+\infty)=2$ and $\mathbf{s r}_{11}=\mathbf{s r}_{0}=0$. But $\mathbf{s r}_{22} \leq 0$ yields 
$\operatorname{MVar}(\mathcal{P} ;-\infty,+\infty) \leq 1$, which is a contradiction. Thus $f(\lambda)=0$ has no positive root and by Lemma 2.1 the two ellipsoids overlap.

Theorem 3.10. Let $f(\lambda)=0$ be the characteristic equation of two ellipsoids $\mathcal{A}: X^{T} A X=0$ and $\mathcal{B}$ : $X^{T} B X=0$.

1. The two ellipsoids $\mathcal{A}$ and $\mathcal{B}$ are separate if and only if $\operatorname{Var}(1, a, b, c, d)=2$ and

(a) $\mathbf{s r}_{22}>0, \mathbf{s r}_{11}>0, \mathbf{s r}_{0}>0$; or

(b) $\mathbf{s r}_{22}>0, \mathbf{s r}_{11}>0, \mathbf{s r}_{10}>0, \mathbf{s r}_{0}=0$.

2. The two ellipsoids $\mathcal{A}$ and $\mathcal{B}$ touch each other externally if and only if

(a) $\mathbf{s r}_{22}>0, \mathbf{s r}_{11}>0, \mathbf{s r}_{10}<0, \mathbf{s r}_{0}=0$; or

(b) $\mathbf{s r}_{22}>0, \mathbf{s r}_{20}<0, \mathbf{s r}_{11}=0, \mathbf{s r}_{0}=0$.

In the other cases, the two ellipsoids overlap.

Proof. 1. " $\Longrightarrow "$ :

Since $\mathcal{A}$ and $\mathcal{B}$ are separate, by Theorem 3.6, $\operatorname{Var}(1, a, b, c, d)=2$ and $f(\lambda)=0$ has four real roots, two of which are distinct positive reals and the other two are negative. This leads to two subcases:

(a) $f(\lambda)=0$ has two distinct negative roots. By Lemma 3.8, we have $\mathbf{s r}_{22}>0, \mathbf{s r}_{11}>0, \mathbf{s r}_{0}>0$;

(b) $f(\lambda)=0$ has one negative double root. By Lemma $3.8, \mathbf{s r}_{22}>0, \mathbf{s r}_{11}>0, \mathbf{s r}_{0}=0$. By Lemma 2.3, $\operatorname{gcd}\left(f(\lambda), f^{\prime}(\lambda)\right)=\mathbf{s r}_{1}=\mathbf{s r}_{11} \lambda+\mathbf{s r}_{10}=\mathbf{s r}_{11}\left(\lambda-\lambda_{0}\right)$, where $\lambda_{0}=-\mathbf{s r}_{10} / \mathbf{s r}_{11}$ is the negative double root of $f(\lambda)=0$. Hence $\mathbf{s r}_{11}>0$ yields $\mathbf{s r}_{10}>0$.

" " :

By Theorem 3.8, both a) and b) indicate that $f(\lambda)=0$ has four real roots. Since $\operatorname{Var}(1, a, b, c, d)=2$, by Theorem 3.6 the two ellipsoids are separate or externally touch.

(a) since $\mathbf{s r}_{0} \neq 0, f(\lambda)=0$ has no multiple root. Hence by Lemma 2.1 the two ellipsoids are separate.

(b) since $\mathbf{s r}_{0}=0$ and $\mathbf{s r}_{11} \neq 0, f(\lambda)=0$ has one double root and two simple roots. The double root $\lambda_{0}$ is the root of $\mathbf{s r}_{1}=\mathbf{s r}_{11} \lambda+\mathbf{s r}_{10}$, and hence negative because $\mathbf{s r}_{11}>0, \mathbf{s r}_{10}>0$. Therefore by Lemma 2.1 the two ellipsoids cannot be externally touching, and are therefore separate.

2. " $\Longrightarrow "$ :

Since $\mathcal{A}$ and $\mathcal{B}$ are externally touching, by Theorem 3.6 $f(\lambda)=0$ has four real roots, two of which are a positive double root and the other two are negative. This also leads to two subcases:

(a) $f(\lambda)=0$ has two distinct negative roots, which by Lemma 3.8 yields $\mathbf{s r}_{22}>0, \mathbf{s r}_{11}>0, \mathbf{s r}_{0}=0$. By Lemma 2.3, $\mathbf{s r}_{1}=\mathbf{s r}_{11} \lambda+\mathbf{s r}_{10}=\operatorname{gcd}\left(f(\lambda), f^{\prime}(\lambda)\right)$ and therefore $\lambda_{0}=-\mathbf{s r}_{10} / \mathbf{s r}_{11}$ is the positive double root of $f(\lambda)=0$. Now since $\mathbf{s r}_{11}>0$, we have $\mathbf{s r}_{10}<0$. 
(b) $f(\lambda)=0$ has one negative double root. By Lemma $3.8 \mathbf{s r}_{22}>0, \mathbf{s r}_{11}=0, \mathbf{s r}_{0}=0$. By Lemma 2.3 $\mathbf{s r}_{2}=\mathbf{s r}_{22} \lambda^{2}+\mathbf{s r}_{21} \lambda+\mathbf{s r}_{20}=\operatorname{gcd}\left(f(\lambda), f^{\prime}(\lambda)\right)=\mathbf{s r}_{22}\left(\lambda-\lambda_{0}\right)\left(\lambda-\lambda_{1}\right)$, where $\lambda_{0}$ is the positive double root and $\lambda_{1}$ is the negative double root. Hence $\mathbf{s r}_{20} / \mathbf{s r}_{22}=\lambda_{0} \lambda_{1}<0$. Since $\mathbf{s r}_{22}>0$, we have $\mathbf{s r}_{20}<0$.

$" \Longleftarrow ":$

(a) by Lemma 3.8, $f(\lambda)=0$ has one double root and two simple roots. By Lemma $2.3, \mathbf{s r}_{1}=$ $\mathbf{s r}_{11} \lambda+\mathbf{s r}_{10}=\operatorname{gcd}\left(f(\lambda), f^{\prime}(\lambda)\right)$ and therefore $\lambda_{0}=-\mathbf{s} \mathbf{r}_{10} / \mathbf{s r}_{11}>0$ is the double root of $f(\lambda)=0$.

By lemma 2.1 the two ellipsoids are externally touching.

(b) by Lemma 3.8 there are two subcases:

i. $f(\lambda)=0$ has two double roots. By Lemma $2.3 \mathbf{s r}_{2}=\mathbf{s r}_{22} \lambda^{2}+\mathbf{s r}_{21} \lambda+\mathbf{s r}_{20}=\operatorname{gcd}\left(f(\lambda), f^{\prime}(\lambda)\right)=$ $\mathbf{s r}_{22}\left(\lambda-\lambda_{0}\right)\left(\lambda-\lambda_{1}\right)$, where $\lambda_{0}$ and $\lambda_{1}$ are the two double roots. Since $\lambda_{0} \lambda_{1}=\mathbf{s r}_{20} / \mathbf{s r}_{22}<0$, one of the double root should be positive. By Lemma 2.1 the two ellipsoids are in external touch.

ii. $f(\lambda)=0$ has one simple root and one triple root $\lambda_{0}$. Then $\mathbf{s r}_{20} / \mathbf{s r}_{22}=\lambda_{0}^{2}>0$, which contradicts the fact that $\mathbf{s r}_{22}>0$ and $\mathbf{s r}_{20}<0$. Hence this subcase never happens.

Remark 3.1. According to Theorem 3.10, the subresultant coefficient $\mathbf{s r}_{10}$ is crucial for distinguishing the two configurations of separation and external touching. Note that Gonzalez-Vega and Mainar (2008) uses principal subresultant sequences (i.e., $\mathbf{s r}_{22}, \mathbf{s r}_{11}, \mathbf{s r}_{0}$ ) to derive the explicit formulae which do not distinguish separation from external touching of two static ellipsoids. We achieves this distinction by considering the sign of $\mathbf{s r}_{10}$.

Table 2 summarizes Theorem 3.10 on characterizing the configuration, i.e., separation, external touching or overlapping, of two static ellipsoids.

\section{Computation Costs}

Our method for determining the configuration of two ellipsoids involves only the evaluation of the explicit formulae $\mathbf{s r}_{22}, \mathbf{s r}_{20}, \mathbf{s r}_{11}, \mathbf{s r}_{10}, \mathbf{s r}_{0}$ and $\operatorname{Var}(1, a, b, c, d)$. Here we adopt the optimized evaluation of these five polynomials provided by Emiris and Tsigaridas (2008) obtained from the Bezoutian matrix. Let

$$
\bar{b}=-\frac{a}{4}, \bar{c}=\frac{b}{6}, \bar{d}=-\frac{c}{4}, \bar{e}=d,
$$




\begin{tabular}{|c|c|c|c|c|c|c|c|c|}
\hline \multirow{2}{*}{\multicolumn{2}{|c|}{ Cases }} & \multirow[b]{2}{*}{$\operatorname{Var}(1, a, b, c, d)$} & \multicolumn{2}{|c|}{$\mathrm{sr}_{2}(\lambda)$} & \multicolumn{2}{|c|}{$\operatorname{sr}_{1}(\lambda)$} & \multirow[b]{2}{*}{$\operatorname{sr}_{0}$} & \multirow{2}{*}{$\begin{array}{c}\text { Ellipsoids } \\
\text { configurations }\end{array}$} \\
\hline & & & $\mathrm{sr}_{22}$ & $\mathrm{sr}_{20}$ & $\operatorname{sr}_{11}$ & $\mathrm{sr}_{10}$ & & \\
\hline$(1)$ & & 2 & $>0$ & & $>0$ & & $>0$ & separate \\
\hline \multirow{2}{*}{ (2) } & & 2 & $>0$ & & $>0$ & $>0$ & $=0$ & separate \\
\hline & & & $>0$ & & $>0$ & $<0$ & $=0$ & externally touch \\
\hline$(3)$ & & & $>0$ & $<0$ & $=0$ & & $=0$ & externally touch \\
\hline \multicolumn{9}{|c|}{ The remaining cases correspond to overlapping ellipsoids. } \\
\hline
\end{tabular}

Table 2: Algebraic conditions for characterizing the configuration of two ellipsoids. The case numbers correspond to that of Lemma 3.8.

and let

$$
\begin{array}{ccc}
\Delta_{2}=\bar{b}^{2}-\bar{c} & W_{1}=\bar{d}-\bar{b} \bar{c} & T=-9 W_{1}^{2}+27 \Delta_{2} \Delta_{3}-3 W_{3} \Delta_{2} \\
\Delta_{3}=\bar{c}^{2}-\bar{b} \bar{d} & W_{2}=\bar{b} \bar{e}-\bar{c} \bar{d} & A=W_{3}+3 \Delta_{3} \\
& W_{3}=\bar{e}-\bar{b} \bar{d} & B=-\bar{d} W_{1}-\bar{e} \Delta_{2}-\bar{c} \Delta_{3} \\
& & T_{2}=A W_{1}-3 \bar{b} B \\
\Delta_{1}=A^{3}-27 B^{2} .
\end{array}
$$

The two explicit formulae are then given by

$$
\mathbf{s r}_{22}:=\Delta_{2}, \mathbf{s r}_{20}:=-W_{3}, \mathbf{s r}_{11}:=T, \mathbf{s r}_{10}:=T_{2}, \mathbf{s r}_{0}:=\Delta_{1}
$$

up to a positive constant multiple. The above expressions take 28 multiplications and 12 additions.

\section{Application: Continuous Collision Detection for Two Moving Ellipsoids}

\subsection{Algorithm}

Let $M(t)$ be a $4 \times 4$ matrix, whose entries are arbitrary smooth functions in $t$, that represents an arbitrary continuous deformation and motion in $\mathbb{R}^{3}$. By applying such deformation and motion to an ellipsoid 
$\mathcal{A}: X^{T} A X=0$, we obtain a moving ellipsoid $\mathcal{A}(t): X^{T} M^{-1}(t)^{T} A M^{-1}(t) X=X^{T} A(t) X=0$. Next we extend our method to solving continuous collision detection for two moving ellipsoids $\mathcal{A}(t): X^{T} A(t) X=0$ and $\mathcal{B}(t): X^{T} B(t) X=0$, whose center positions vary and shapes deform with respect to a continuously varied parameter $t \in[0,1]$. The two moving ellipsoids $\mathcal{A}(t)$ and $\mathcal{B}(t)$ are said to be collision-free if $\mathcal{A}(t)$ and $\mathcal{B}(t)$ are separate for all $t \in[0,1]$; otherwise $\mathcal{A}(t)$ and $\mathcal{B}(t)$ collide.

In this continuous setting, the characteristic polynomial associated with $\mathcal{A}(t)$ and $\mathcal{B}(t)$ becomes a bivariate polynomial both in parameter $\lambda$ and parameter $t$ which is given by $f(\lambda ; t)=\operatorname{det}(\lambda A(t)+B(t))$. Clearly, $f(\lambda ; t)$ is of degree 4 in $\lambda$ with coefficients as functions of $t$. We divide $f(\lambda ; t)$ by its leading coefficient in $\lambda$ and get

$$
f(\lambda ; t)=\lambda^{4}+a(t) \lambda^{3}+b(t) \lambda^{2}+c(t) \lambda+d(t)
$$

where $a(t), b(t), c(t), d(t)$ are all functions in $t$. We shall study the subresultants $\mathbf{s r}_{0}(t)$ and $\mathbf{s r}_{i}(\lambda ; t), i=1,2$ of $f(\lambda ; t)$ and $f_{\lambda}(\lambda ; t)$, and denote the coefficient of the degree $j$ term in $\lambda$ of the polynomial $\mathbf{s r}_{i}(\lambda ; t)$ by $\mathbf{s r}_{i j}(t), i=1,2, j=0, \ldots, \operatorname{deg}\left(\mathbf{s r}_{i}\right)$.

Theorem 5.1. Let $0 \leq t_{1}<t_{2}<\cdots<t_{m} \leq 1, m \geq 0$, be all the distinct contact time instants in $[0,1]$ at which two given moving ellipsoids are in external touch. Let $t_{0}=0, t_{m+1}=1$ and let $\delta_{i}$ be an arbitrary number in interval $\left(t_{i}, t_{i+1}\right), i=0, \cdots, m$. Then the configuration of the two moving ellipsoids (i.e., whether they overlap or are separate) does not change during time interval $\left(t_{i}, t_{i+1}\right)$, and therefore can be decided by their configuration at the time instant $t=\delta_{i}, i=0, \ldots, m$.

Proof. Since the center positions and the shapes of the two moving ellipsoids vary continuously, if the configuration of two ellipsoids changes from separation to overlapping, or vice versa, there must be a contact time instant $t^{*}$ at which the ellipsoids are in external touch. Therefore, within a time interval $\left(t_{i}, t_{i+1}\right)$ that does not contain any other contact time instants, the configuration of the ellipsoids remains the same. Hence we need only check the status of the two moving ellipsoids at a time instant $\delta_{i} \in\left(t_{i}, t_{i+1}\right)$ to decide their configuration during the entire time interval $\left(t_{i}, t_{i+1}\right)$.

We can see from the above theorem that the primary task for continuous collision detection for two moving ellipsoids is to determine the so-called contact time instants at which the ellipsoids are touching externally. The configuration of the ellipsoids in between two contact time instants can then be easily identified using the algebraic conditions as established in Theorem 3.10.

Next, we explain how these contact time instants can be found.

Theorem 5.2. Suppose that $\mathbf{s r}_{0}(t) \not \equiv 0$. If two ellipsoids $\mathcal{A}(t)$ and $\mathcal{B}(t)$ touch externally at $t_{0}$, we have $\mathbf{s r}_{0}\left(t_{0}\right)=0$.

Proof. This follows immediately from Theorem 3.10. 
Note 1 . The special situation $\mathbf{s r}_{0}(t) \equiv 0$ happens when the characteristic equation $f(\lambda ; t)=0$ always has a double root $\lambda(t)$ for any time instant $t \in[0,1]$, i.e., $f(\lambda ; t)=\left(\lambda-\lambda^{*}(t)\right)^{2} \tilde{f}(\lambda ; t)$. Geometrically this occurs when two moving ellipsoids, at every time instant $t$, touch internally or externally, or have a reducible intersection in $\mathbb{C}^{3}$ (see Tu et al. (2009) and Example 5.1).

Lemma 5.3. Given two moving ellipsoids $\mathcal{A}(t)$ and $\mathcal{B}(t), t \in[0,1]$, we have either one of the following cases:

1. $\mathcal{A}(t)$ and $\mathcal{B}(t)$ are in external touch for all $t \in[0,1]$;

2. $\mathcal{A}(t)$ and $\mathcal{B}(t)$ are in external touch at some discrete time instants $t_{i} \in[0,1]$;

3. $\mathcal{A}(t)$ and $\mathcal{B}(t)$ never touch during $[0,1]$.

Proof. Suppose that $\mathcal{A}(t)$ and $\mathcal{B}(t)$ touch externally at all $t$ within a time span $\left[t_{0}, t_{1}\right] \subset[0,1]$. Then their characteristic equation $f(\lambda ; t)=0$ has a positive double root in $\lambda$ (Theorem 2.1) for all $t \in\left[t_{0}, t_{1}\right]$. Due to the continuity of the roots of a polynomial (see e.g., Bhatia (1997)), the roots of $f(\lambda ; t)=0$ can be expressed as complex-valued polynomials $\alpha_{i}(t)$, where $i=1,2,3,4$. Without loss of generality, let $\alpha_{1}(t) \equiv \alpha_{2}(t)>0$ be the double root for $t \in\left[t_{0}, t_{1}\right]$. Since $\alpha_{1}(t)$ and $\alpha_{2}(t)$ are polynomials, we must have $\alpha_{1}(t) \equiv \alpha_{2}(t)>0$ for $t \in[0,1]$, which means that the moving ellipsoids are always in external touch. Hence, the moving ellipsoids can either be in external touch for all $t \in[0,1]$ or be in external touch only at some (if there is any) discrete contact time instants.

Theorem 5.4. Suppose that $\mathbf{s r}_{0}(t) \equiv 0$. If the moving ellipsoids $\mathcal{A}(t)$ and $\mathcal{B}(t)$ are in external touch only at some discrete contact time instants $t_{i} \in[0,1]$, where $i=1, \ldots, n$ and $n \geq 0$, then at each $t_{i}$, we have $\mathbf{s r}_{11}\left(t_{i}\right)=0$.

Proof. Since $\mathbf{s r}_{0}(t) \equiv 0$, we have $f(\lambda ; t)=\left(\lambda-\lambda^{*}(t)\right)^{2} \tilde{f}(\lambda ; t)$ (Note 1$)$. Since $\mathcal{A}(t)$ and $\mathcal{B}(t)$ are not in external touch for all $t, \lambda^{*}(t)$ cannot be a positive double root. Also, since $\lambda=0$ cannot be a root of the characteristic equation $f(\lambda ; t)=0$ for any $t$, by continuity of the roots of a polynomial Bhatia (1997), $\lambda^{*}(t)<0$ for all $t$. Now, consider at a contact time instant $t_{i}, f(\lambda ; t)=0$ has an additional positive double root and therefore $f\left(\lambda ; t_{i}\right)=0$ has two double roots. By Lemma 3.8, we therefore have $\mathbf{s r}_{11}\left(t_{i}\right)=0$.

Note 2. Consider two moving spheres that are in external touch at only some discrete contact time instants. Since they always have a reducible intersection in $\mathbb{C}^{3}$, no matter whether they are separate or not, their characteristic equation always contains a double root (which is negative). Hence, $\mathbf{s r}_{0}(t) \equiv 0$. Furthermore, $\mathbf{s r}_{11}\left(t_{i}\right)=0$ if the two spheres are in external touch at $t_{i}$.

Theorem 5.5. Suppose that $\mathbf{s r}_{0}(t) \equiv 0$ and $\mathbf{s r}_{11}(t) \not \equiv 0$. Let $t_{1}, t_{2}, \ldots, t_{n}, n \geq 0$, be all the distinct real roots of $\mathbf{s r}_{11}(t)=0$ in $[0,1]$. Let $\delta$ be an arbitrary number in $[0,1] \backslash\left\{t_{1}, t_{2}, \ldots, t_{n}\right\}$. If the ellipsoids $\mathcal{A}(\delta)$ and $\mathcal{B}(\delta)$ touch externally, then $\mathcal{A}(t)$ and $\mathcal{B}(t)$ are in external touch throughout $[0,1]$. 
Proof. This follows from Lemma 5.3 and Theorem 5.4.

Theorem 5.6. Suppose that $\mathbf{s r}_{0}(t) \equiv 0$ and $\mathbf{s r}_{11}(t) \equiv 0$. Then either the two ellipsoids $\mathcal{A}(t)$ and $\mathcal{B}(t)$ touch externally throughout $[0,1]$, or they do not have any external touch at all in $[0,1]$.

Proof. Since $\mathbf{s r}_{0}(t) \equiv 0$ and $\mathbf{s r}_{11}(t) \equiv 0$, by Lemma 3.8, $f(\lambda ; t)=0$ always have two double roots or one quadruple root in $\lambda$. Due to the continuity of the roots of a polynomial (see e.g., Bhatia (1997)) and the fact that $\lambda=0, \lambda= \pm \infty$ are not roots of $f(\lambda ; t)=0$, the signs of the roots remain the same in $[0,1]$. Now, if $f\left(\lambda ; t_{i}\right)=0$ has a positive double root $\lambda_{0}\left(t_{i}\right)$ for some $t_{i} \in[0,1]$, then $\lambda_{0}(t)$ remains positive throughout $[0,1]$, which means that $\mathcal{A}(t)$ and $\mathcal{B}(t)$ are in external touch throughout $[0,1]$. On the other hand, if $f(\lambda ; t)=0$ does not have a positive double root for any $t \in[0,1]$, then $\mathcal{A}(t)$ and $\mathcal{B}(t)$ do not touch externally at all in the interval.

Note 3. Theorem 5.6 implies that when $\mathbf{s r}_{0}(t) \equiv 0$ and $\mathbf{s r}_{11}(t) \equiv 0$, the configuration of the ellipsoids throughout the time span can be determined by their configuration at any particular time instant, e.g., at $t=0$.

We now summarize the above analysis in Algorithm 1 for continuous collision detection for two moving ellipsoids. Using Theorems 5.2, 5.4, 5.5 and 5.6, we obtain a set $Z$ of time instants which captures all the contact time instants of two moving ellipsoids by solving for the roots of some functions under different conditions. The set $Z$ may also contain other time instants not corresponding to any contact, which can be eliminated easily by checking against the algebraic conditions given by Theorem 3.10. Again by Theorem 3.10, the configuration of the ellipsoids at each interval defined by two consecutive contact time instants can then be determined. 


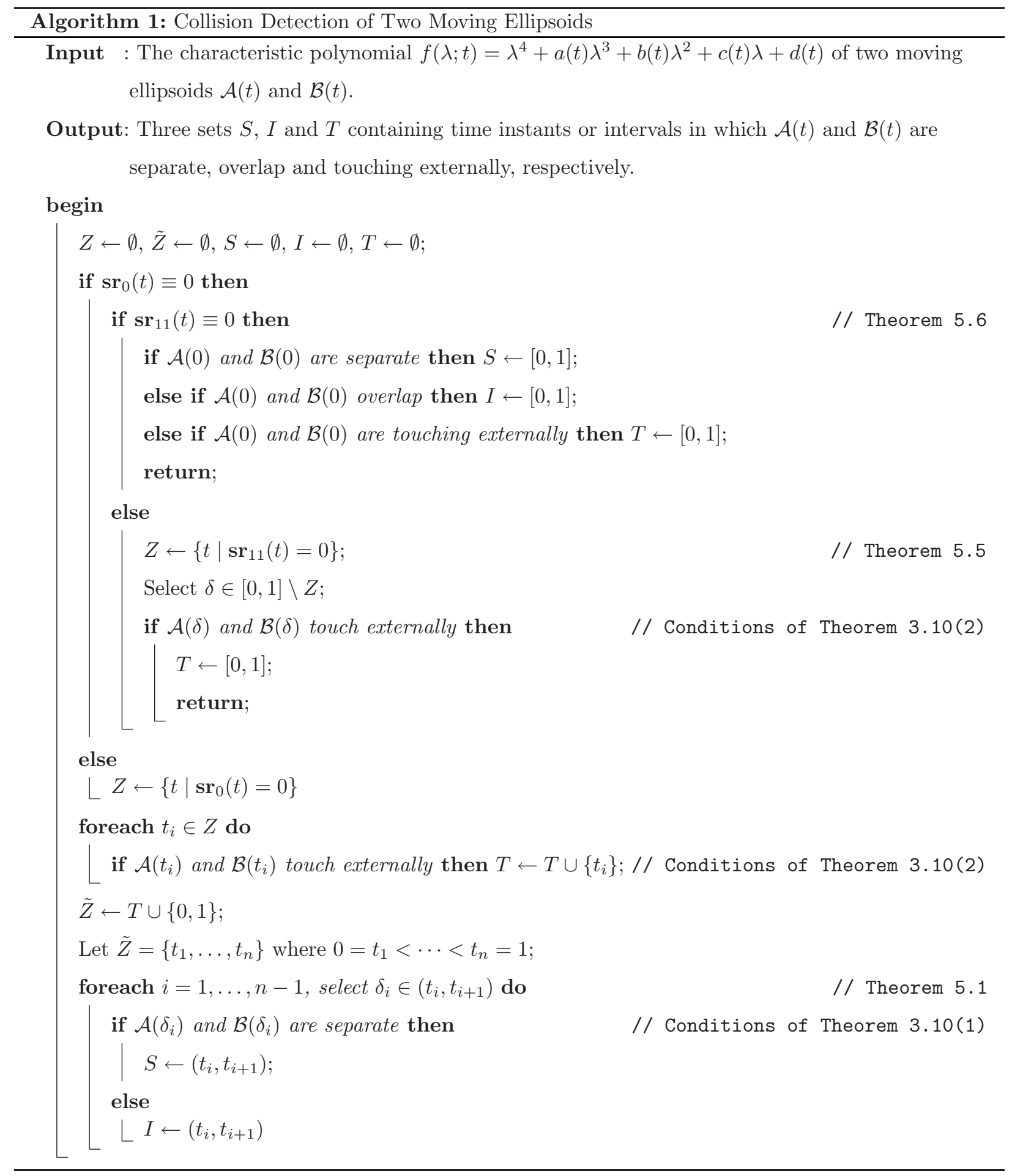




\subsection{Examples}

Example 5.1. Let $\mathcal{A}(t)$ and $\mathcal{B}(t)$ be two moving ellipsoids defined by

$$
\frac{(x+12 t-11)^{2}}{4}+y^{2}+z^{2}=1 \quad \text { and } \quad \frac{(x-3)^{2}}{4}+(y-4 t+2)^{2}+(z-4 t+4)^{2}=1,
$$

respectively, where $t \in[0,1]$. The characteristic polynomial associated with $\mathcal{A}(t)$ and $\mathcal{B}(t)$ is

$$
\begin{aligned}
f(\lambda ; t) & =\lambda^{4}+\left(-68 t^{2}+96 t-32\right) \lambda^{3}+\left(-136 t^{2}+192 t-66\right) \lambda^{2}+\left(-68 t^{2}+96 t-32\right) \lambda+1 \\
& =-(\lambda+1)^{2}\left(-\lambda^{2}+\left(68 t^{2}-96 t+34\right) \lambda-1\right)
\end{aligned}
$$

up to a constant multiple. Since $\mathbf{s r}_{0}(t) \equiv 0$, we shall next compute

$$
\begin{aligned}
\mathbf{s r}_{11}(t) & =42762752 t^{8}-241483776 t^{7}+599418368 t^{6}-854175744 t^{5} \\
& +764204544 t^{4}-439492608 t^{3}+158630400 t^{2}-32845824 t+2985984 .
\end{aligned}
$$

Solving the real roots for $\mathbf{s r}_{11}(t)$ in $[0,1]$, we obtain $t_{1}=0.5395042868, t_{2}=0.8722604191$ which are both confirmed to be the contact time instants of the ellipsoids by checking against the conditions in Theorem 3.10. Selecting

$$
\delta_{0}=0.2 \in\left(0, t_{1}\right), \quad \delta_{1}=0.7 \in\left(t_{1}, t_{2}\right), \quad \delta_{2}=0.95 \in\left(t_{2}, 1\right),
$$

and by checking the collision states at $t=\delta_{0}, \delta_{1}, \delta_{2}$, we conclude that the two ellipsoids are separate during time interval $\left[0, t_{1}\right)$, overlap during $\left(t_{1}, t_{2}\right)$, and are separate again during $\left(t_{2}, 1\right]$ (Figure 1$)$.

The following example shows that our approach not only works for rational motions but also allows arbitrary functional motions, e.g., helical motions, of two moving ellipsoids.

Example 5.2. Let $\mathcal{A}$ and $\mathcal{B}$ be two static ellipsoids defined by

$$
x^{2}+\frac{y^{2}}{4}+z^{2}=1 \quad \text { and } \quad x^{2}+y^{2}+\frac{(z-5)^{2}}{9}=1,
$$

respectively. Let $\mathcal{A}(t)$ be a moving ellipsoid defined by applying to $\mathcal{A}$ a rotation about the axis $(1,0,0)^{T}$ by an angle $10 t$ and then a translation along the helical curve $P(t)=(\cos 10 t, \sin 10 t, 10 t)$, where $t \in[0,1]$. The coefficients of the characteristic polynomial associated with the moving ellipsoid $\mathcal{A}(t)$ and the static ellipsoid $\mathcal{B}$ are functions in $t$ that contains trigonometric terms; the expressions are long and hence are omitted here.

We solve the transcedental function $\mathbf{s r}_{0}(t)$ by the simple bracketing and bisection method. Other methods, such as the secant method, can also be used for root finding (see Press et al. (2007) for more details). The real roots of $\mathbf{s r}_{0}(t)$ in $[0,1]$ are found to be $t_{1}=0.0749830692$ and $t_{2}=0.8913371204$, which are both 


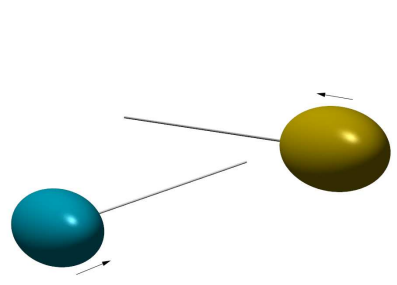

(a) $t=0$

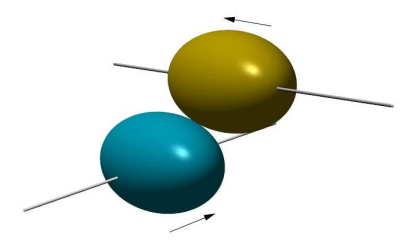

(b) $t=t_{1}=0.54$

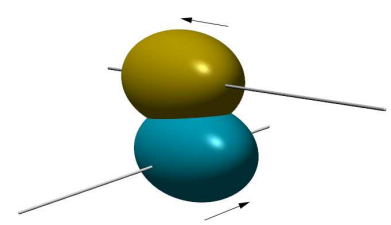

(c) $t=\delta_{1}=0.7$

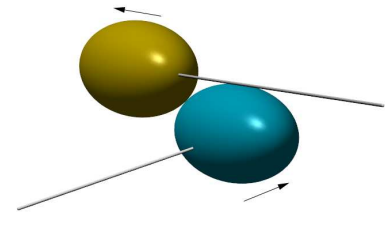

(d) $t=t_{2}=0.87$

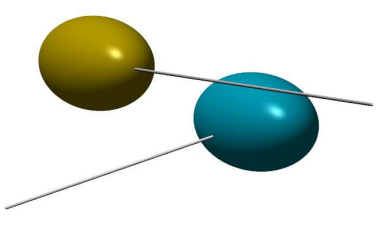

(e) $t=1$

Figure 1: Configurations of two moving ellipsoids under rational motion in Example 5.1. The ellipsoids touch externally at $t=t_{1}$ and $t=t_{2}$.

confirmed to be the contact time instants of the ellipsoids. We check the collision states at

$$
\delta_{0}=0.02 \in\left(0, t_{1}\right), \quad \delta_{1}=0.4 \in\left(t_{1}, t_{2}\right), \quad \delta_{2}=0.95 \in\left(t_{2}, 1\right),
$$

and conclude that the two ellipsoids are separate in $\left[0, t_{1}\right)$, overlap in $\left(t_{1}, t_{2}\right)$, and are separate again in $\left(t_{2}, 1\right]$ (Figure 2).

\subsection{Comparison}

In this section, we compare our method with Choi et al. (2009) on continuous collision detection for two ellipsoids. Since the method in Choi et al. (2009) only deals with ellipsoids under rational motions, the examples we use here are also confined to rational motions. Both algorithms are implemented in $\mathrm{C}++$ and the tests are run on a workstation with an Intel Xeon 3.33-GHz CPU. Double precision floating-point arithmetic is used for all computations in the comparison. Polynomials are represented in the Bernstein form in order to improve the robustness and accuracy of the computations. Root solving of polynomials are then done by subdivision using the de Casteljau algorithm. The algorithms are applied to three pairs of moving ellipsoids under different motion types to detect their collision states over a specific time span and the performance of the algorithms are listed in Table 3. Each test is run for 1,000 times and the average running time is taken.

The degree 2 rational rigid motion includes the simple yet commonly used motion in which an object assumes a degree 2 rotation plus a linear translation. Under this kind of rigid motion, the two methods 


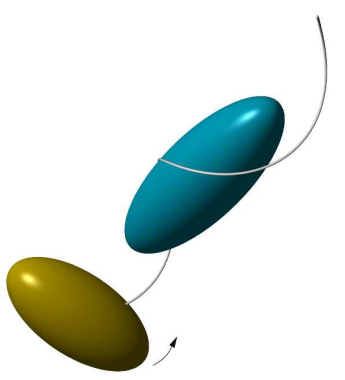

(a) $t=0$

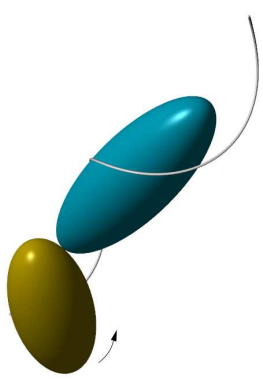

(b) $t=t_{1}=0.07$

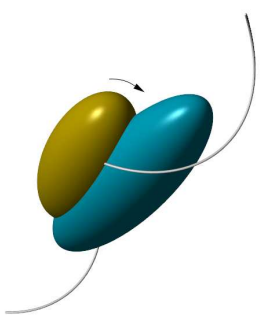

(c) $t=\delta_{1}=0.45$

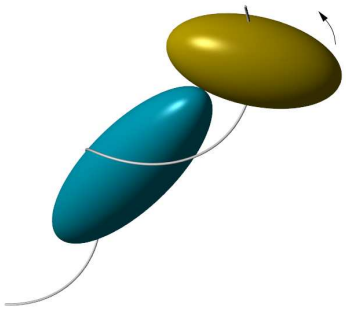

(d) $t=t_{2}=0.89$

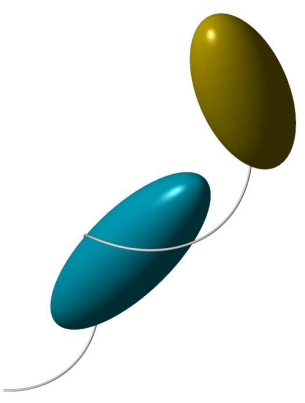

(e) $t=1$

Figure 2: Configurations of two ellipsoids, one static and the other moves under non-rational helical motion, in Example 5.2. The ellipsoids touch externally at $t=t_{1}$ and $t=t_{2}$.

have comparable performances and can both complete CCD in about $0.1 \mathrm{~ms}$. The next pair of ellipsoids we study are under degree 2 rational affine motion, that is, one with deformation. The degree of $f(\lambda ; t)$ in $t$ is 36 in this particular example. The method in Choi et al. (2009) needs much longer (about $2.5 \mathrm{~ms}$ ) to compute CCD, because their method deals with a bivariate function $f(\lambda ; t)$ and basically needs to find a pathway $\lambda(t)$ such that $f(\lambda ; t)>0$ for all $t$ to declare that the ellipsoids are always separate. The time taken therefore depends not only on the degree of the motion but also on the topology of the zero set of $f(\lambda ; t)$. In this example, the two moving ellipsoids are in close proximity from time to time but remain separate within the entire time span. The approach in Choi et al. (2009) therefore takes longer to find the pathway $\lambda(t)$. On the other hand, our method does not depend on the complexity of $f(\lambda ; t)$ and can solve the CCD in 0.7 ms.

In the last example, the moving ellipsoids are under rational motions of degree 4 with large deformations (Example 2 of Choi et al. (2009)). The degree of $f(\lambda ; t)$ in $t$ is 48 . The Choi et al. (2009) method takes about $1 \mathrm{~ms}$ while ours takes about $13 \mathrm{~ms}$ to complete CCD. The slower performance of our method is due to the high degree in the subresultant expressions. The degree of $\operatorname{sr}_{0}(t)$ is 288 ; its composition and root finding are therefore time consuming. When only the first contact time instant of two moving ellipsoids is required 


\begin{tabular}{|c|c|c|c|c|c|c|c|}
\hline \multirow{2}{*}{ Motion Type } & \multirow{3}{*}{$\operatorname{deg}_{t}(f(\lambda ; t))$} & \multirow{2}{*}{$\operatorname{deg}\left(\operatorname{sr}_{0}(t)\right)$} & \multicolumn{6}{|c|}{ Time $(\mathrm{ms})$} \\
\cline { 4 - 7 } & & & Set up & \multicolumn{2}{|c|}{ Solve $f(\lambda ; t)$} & \multicolumn{2}{c|}{ Total for CCD } \\
\cline { 4 - 7 } & & & $f(\lambda ; t)$ & Choi 2009 & Our & Choi 2009 & Our \\
\hline degree 2 rigid & 4 & 24 & 0.014 & 0.094 & 0.096 & 0.108 & 0.11 \\
degree 2 affine & 36 & 216 & 0.12 & 2.411 & 0.571 & 2.531 & 0.691 \\
degree 4 affine & 48 & 288 & 0.202 & 0.965 & 12.707 & 1.167 & 12.909 \\
\hline
\end{tabular}

Table 3: Run-time performance of our method against Choi et al. (2009) to solve CCD of two moving ellipsoids under rational motions. The timing under the column "Solving $f(\lambda ; t)$ " is the time taken for determining the collision status over a given time span. The total time for CCD is the sum of the time taken for setting up and solving $f(\lambda ; t)$. All timings are averaged over 1,000 runs.

\begin{tabular}{|c|c|c|c|c|c|}
\hline & & & \multicolumn{3}{|c|}{ Time $(\mathrm{s})$} \\
\cline { 4 - 6 } Motion Type & $\operatorname{deg}_{t}(f(\lambda ; t))$ & $\operatorname{deg}\left(\operatorname{sr}_{0}(t)\right)$ & Set up $f(\lambda ; t)$ & Solve $f(\lambda ; t)$ & Total for CCD \\
\hline degree 2 rigid & 4 & 24 & 0.002169 & 0.016 & 0.018169 \\
degree 2 affine & 36 & 216 & 0.002683 & 1.326 & 1.328683 \\
\hline
\end{tabular}

Table 4: Run-time performance of our method to symbolically solve CCD of two moving ellipsoids under rational motions. All timings are averaged over 1,000 runs.

(which is a common output for CCD), our method does not need to solve for all roots of the subresultants and can complete CCD in $0.4 \mathrm{~ms}$.

We remark here that the above examples serve to demonstrate the efficiency of our method when time performance is of major concern. Both Choi et al. (2009) and ours are exact continuous collision detection methods in the sense that no discretization of the time domain is needed. However, as we mentioned in Section 1, Choi et al. (2009) solve a bivariate characteristic equation using numerical computations. We therefore use a float-point implementation of our method for comparison with Choi et al. (2009). Note that, when high numerical accuracy is desired, our method has the advantage that exact arithmetic can be used to achieve any required accuracy. We also listed in Table 4 the corresponding time costs for the same collision detection examples under degree 2 rigid motion and degree 2 affine motion using symbolic computation.

\section{Conclusions}

We use five explicit formulae to decide the geometric configurations, that are, separate, externally touching or overlapping of two ellipsoids. Our derivation is based on the algebraic conditions provided in Wang et al. (2001), which shows the correspondence between the root patterns of the characteristic polynomial and the configurations of two ellipsoids. The explicit formulae are composed of the coefficients of the subresultant sequence of the characteristic polynomial and its first derivative. These algebraic formulae can be applied naturally to continuous collision detection for two moving ellipsoids whose relative positions as well as shapes vary along time governed by arbitrary continuous functions. In future work, we expect to apply simple techniques and develop explicit formulae to determine the relative geometric configuration of two general quadrics. 


\section{References}

Basu, S., Pollack, R., Roy, M.F., 2006. Algorithms in Real Algebraic Geometry (Algorithms and Computation in Mathematics). Springer-Verlag New York, Inc., Secaucus, NJ, USA.

Bhatia, R., 1997. Matrix Analysis. volume 169 of Graduate Texts in Mathematics. Springer-Verlag, New York.

Bouville, C., 1985. Bounding ellipsoids for ray-fractal intersection, in: SIGGRAPH '85: Proceedings of the 12th annual conference on Computer graphics and interactive techniques, ACM Press, New York, NY, USA. pp. 45-52.

Choi, Y.K., Chang, J.W., Wang, W., Kim, M.S., Elber, G., 2009. Continuous collision detection for ellipsoids. IEEE Transactions on Visualization and Computer Graphics 15, 311-325.

Choi, Y.K., Wang, W., Kim, M.S., 2003. Exact collision detection of two moving ellipsoids under rational motions, in: Proc. IEEE Conf. Robot. Autom., Taipei, Taiwan. pp. 349-354.

Choi, Y.K., Wang, W., Liu, Y., Kim, M.S., 2006. Continuous collision detection for two moving elliptic disks. IEEE Transactions on Robotics 22, 213-224.

Eberly, D.H., 2001. 3D Game Engine Design. Academic Press.

Emiris, I.Z., Tsigaridas, E.P., 2008. Real algebraic numbers and polynomial systems of small degree. Theoretical Computer Science 409, 186-199

von zur Gathen, J., Gerhard, J., 1999. Modern computer algebra. Cambridge University Press, New York.

Geddes, K., Czapor, S., Labahn, G., 1992. Algorithms for Computer Algebra. Kluwer Academic Publishers, Norwell, Massachusetts.

Gonzalez-Vega, L., Mainar, E., 2008. Solving the separation problem for two ellipsoids involving only the evaluation of six polynomials (extended abstract), in: Milestones in Computer Algebra (MICA).

Ju, M., Liu, J., Shiang, S., Chien, Y., Hwang, K., Lee, W., 2001. A novel collision detection method based on enclosed ellipsoid, in: Proceedings of 2001 IEEE Conference on Robotics and Automation, pp. 21-26.

Kerber, M., 2009. Division-free computation of subresultants using Bezout matrices. International Journal of Computer Mathematics 86, 2186-2200.

Kumar, P., Yildirim, E., 2005. Minimum-volume enclosing ellipsoids and core sets. Journal of Optimization Theory and Applications 126, 1-21. Cited By (since 1996) 31

Lu, L., Choi, Y.K., Wang, W., Kim, M.S., 2007. Variational 3d shape segmentation for bounding volume computation. Computer Graph Forum 26, 329-338.

Press, W.H., Teukolsky, S.A., Vetterling, W.T., Flannery, B.P., 2007. Numerial Recipes 3rd Edition: The Art of Scientific Computing. Cambridge University Press.

Redon, S., Kheddar, A., Coquillart, S., 2002. Fast continuous collision detection between rigid bodies. Comput. Graph. Forum $21,279-288$.

Rimon, E., Boyd, S., 1997. Obstacle collision detection using best ellipsoid fit. Journal of Intelligent and Robotic Systems 18, 105-126.

Schröker, H.P., 2008. Uniqueness results for minimal enclosing ellipsoids. Computer Aided Geometric Design 25, 756 - 762. Classical Techniques for Applied Geometry.

Shiang, S., Liu, J., Chien, Y., 2000. Estimate of minimum distance between convex polyhedra based on enclosed ellipsoids, in: Proceedings of the IEEE/RST International Conference on Intelligent Robots and Systems, Takamatsu, Japan, October, pp. 739-744.

Teschner, M., Kimmerle, S., Heidelberger, B., Zachmann, G., Raghupathi, L., Fuhrmann, A., Cani, M.P., Faure, F., Magnenat- 
Thalmann, N., Strasser, W., Volino, P., 2005. Collision detection for deformable objects. Comput. Graph. Forum 24, 61-81.

Todd, M.J., Yildirim, E.A., 2007. On khachiyan's algorithm for the computation of minimum-volume enclosing ellipsoids. Discrete Applied Mathematics 155, 1731 - 1744.

Tu, C., Wang, W., Mourrain, B., Wang, J., 2009. Using signature sequence to classify intersection curves of two quadrics. Computer Aided Geometric Design 26, 317-335.

Wang, W., Choi, Y.K., Chan, B., Kim, M.S., Wang, J., 2004. Efficient collision detection for moving ellipsoids using separating planes. Computing 72, 235-246.

Wang, W., Wang, J., Kim, M.S., 2001. An algebraic condition for the separation of two ellipsoids. Comput. Aided Geom. Des. $18,531-539$

Welzl, E., 1991. Smallest enclosing disks (balls and ellipsoids), in: Maurer, H. (Ed.), New Results and New Trends in Computer Science. Springer Berlin / Heidelberg. volume 555 of Lecture Notes in Computer Science, pp. 359-370. 10.1007/BFb0038202. 